This is a postprint version of the following published document:

N. Encinas, M. Pantoja, M. Torres-Remiro, and M.A. Martínez. Approaches to poly(tetrafluoroethylene) adhesive bonding, Presented in part at the 4th International Conference on Advanced Computational Engineering and Experimenting (ACE X 2010), Paris, France, 89 July 2010. The journal of adhesion, 87 (2011), pp. 709-719

DOI: $\underline{10.1080 / 00218464.2011 .596778}$

(C) Taylor \& Francis Group, LLC (2011) 


\title{
Approaches to Poly(Tetrafluoroethylene) Adhesive Bonding
}

\author{
N. Encinas, M. Pantoja, M. Torres-Remiro, and \\ M. A. Martínez \\ Department of Science and Engineering of Materials and Chemical \\ Engineering, IAAB Group Behaviour in Service of Materials, \\ Universidad Carlos III de Madrid, Leganés, Spain
}

In this work, we present an approach to achieve improved adhesive bonding with a poly(tetrafluoroethylene) (PTFE) substrate Surfaces were modified by abrasion, atmospheric air plasma torch (APPT) treatment, and by immersion in basic $(\mathrm{NaOH})$ and strongly acidic/oxidizing $\left(\mathrm{HNO}_{3} / \mathrm{KMnO}_{4}\right)$ solutions. The wetting properties of the polymer were studied in terms of surface energy, and adhesion tests were carried out using polyurethane, acrylic, and epoxy adhesives. The sur face characterisation included surface energy calculation through contact angle measurements, infrared spectroscopy (FTIR ATR), scanning electron microscopy (SEM), and $X$ ray electron diffraction (EDX). Adhesion was evaluated by pull off tests following the UNE EN 24624 standard. Experiments revealed that both oxi dation and plasma treatment enhanced surface energy, defluorination, and the creation of a rougher PTFE surface, resulting in adhesion. Simple oxidation and its combination with plasma treatments yielded the higher tensile strength results, with epoxy as the most suitable adhesive among thase studied. Samples presented adhesive or mixed type failure modes.

Keywords: Adhesion; Atmospheric plasma; Poly(tetrafluoroethylene); Polymeric pre treatments; Surface energy; Wettability

Presented in part at the 4th International Conference on Advanced Computational Engineering and Experimenting (ACE X 2010), Paris, France, 89 July 2010.

Address correspondence to N. Encinas, Department of Science and Engineering of Materials and Chemical Engineering, IAAB Group Behaviour in Service of Materials, Universidad Carlos III de Madrid, Av. Universidad, 30, 28911 Leganés, SPAIN. E mail: nencinas@ing.uc3m.es 


\section{INTRODUCTION}

Poly(tetrafluoroethylene) (PTFE) presents, as other fluoropolymers, unique properties such as mechanical and chemical resistance due to the strong C-F and C-C bonds present in the backbone, good thermal stability (from -196 to $370^{\circ} \mathrm{C}$ ), high ohmic resistance, and low friction coefficient [1,2]. However, the industrial applications of PTFE are hindered because of its low wettability towards both polar and non-polar liquids (Fig. 1) and its poor adhesion properties [3,4]. Thus, modification of its surface properties is a requirement prior to adhesive bonding. Different methods have been traditionally employed, such as wet chemical treatments, radio-frequency, particle beam irradiation, graft polymerization, plasma modification, treative with sodium in liquid ammonia, or photochemistry [5-8]. These processes create a more wettable PTFE surface thanks to the C-F, C-C bonding scission, and $\mathrm{C}-\mathrm{O}, \mathrm{C}=\mathrm{O}, \mathrm{C}=\mathrm{C}$ formation.

In the present work, we modified the PTFE surface in order to improve polarity, thus wettability and adhesion, by subjecting it to to mechanical abrasion, APPT [9], and two types of wet chemical processes: immersion in saturated aqueous $\mathrm{NaOH}$ solution and oxidation with a $\left(\mathrm{KMnO}_{4}+\mathrm{HNO}_{3}\right)$ mixture [10]. The changes in wettability were investigated by contact angle measurements. Compositional and morphological variations were characterized by FTIR-ATR and SEM, respectively. A pull-off test was used to evaluate the improvement in adhesion with acrylic, epoxy, and polyurethane adhesives. Results showed the effectiveness of oxidation and plasma processes to provoke the scission of C-F bonds and the insertion of oxygen- or nitrogencontaining polar functionalities. This mechanism resulted in the improvement of PTFE adhesion properties.

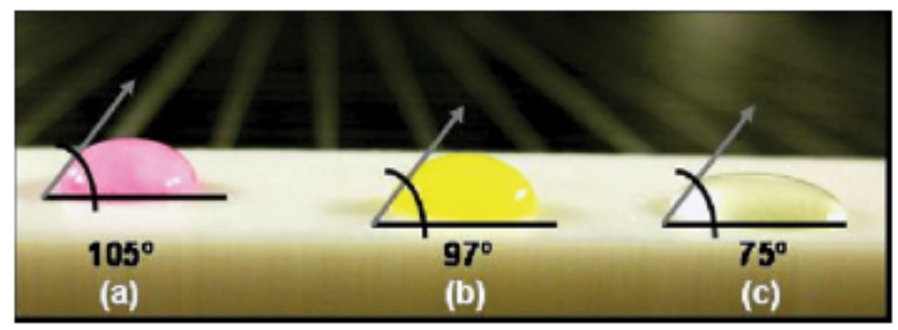

FIGURE 1 Drops of (a) deionised water $\left(\mathrm{H}_{2} \mathrm{O}\right)$, (b) glycerol $\left(\mathrm{C}_{3} \mathrm{H}_{8} \mathrm{O}_{3}\right)$, and (c) diiodomethane $\left(\mathrm{CH}_{2} \mathrm{I}_{2}\right)$ on untreated PTFE sample. Liquids form high contact angles, thus low PTFE wetting (color figure provided online). 


\section{EXPERIMENTAL PROCEDURE}

\subsection{Samples-Preparation}

PTFE samples (Ketersa, Pinto, Madrid, Spain) $(80 \times 20.75 \times 10.5 \mathrm{~cm})$ were properly cleaned with methylethylketone (MEK) and stored until treatment at atmospheric dust-free conditions $\left(25^{\circ} \mathrm{C}, 34 \%\right.$ relative humidity, RH). For the abrasion treatment, a sandpaper of 180 grain size (S180) was selected. Pieces were cleaned again with MEK after abrasion in order to eliminate any contaminating particles. The plasma treatment was carried out with a Plasma Treat $\mathrm{GmbH}$ (Steinhagen, Germany) device operating at a frequency of $17 \mathrm{kHz}$ and a tension discharge of $20 \mathrm{kV}$, with a rotating torch ending in a nozzle (1900 rpm) through which the plasma was expelled. The system contained an electronically speed-controlled platform where the samples were placed, setting a torch-sample distance of $6 \mathrm{~mm}$. The speed of the platform was set at $0.17 \mathrm{~m} / \mathrm{s}$. For the chemical treatments, two conditions were tested: (1) base attack by immersion in a $\mathrm{NaOH} 50 \mathrm{wt} . \%$ solution for $5 \mathrm{~h}$ at $60^{\circ} \mathrm{C}$ and (2) oxidation with a $\left(\mathrm{HNO}_{3} 64 \mathrm{wt} . \%+\mathrm{KMnO}_{4} 6.02 \mathrm{wt} . \%\right)$ mixture by submerging the samples during $5 \mathrm{~h}$ at $100^{\circ} \mathrm{C}$, as Wang et al. previously reported [10].

\subsection{Sample Characterization}

The study of the contact angle formed by different reference liquids onto both pristine and treated PTFE allowed the calculation of the surface energy by the Owens-Wendt-Rabel-Kaelble (OWRK) method, which considers the energy of a solid surface as a sum of a polar and a dispersion component. An OCA 15 plus goniometer (DataPhysics, Neurtek Instruments, Eibar, Guipúzcoa, Spain) described elsewhere [11] provided these results, following the UNE EN-828:2009 standard with an experimental error of $\pm 2{ }^{\circ}$. Six drops per sample and condition were measured, using five different test liquids (deionised water, glycerol, diiodomethane, nitromethane, and 1,5-pentanediol).

A Brucker Tensor 27 (Brucker Optik GmbH, Madrid, Spain) Fourier transform infrared (FTIR) spectrometer was used in the attenuated total multiple reflection mode (ATR) for the evaluation of the compositional changes of the outer surface (5-10 $\mu \mathrm{m}$ depth) of the specimens, recorded from 600 to $4000 \mathrm{~cm}^{1}$. A diamond prism and an incident radiation angle of $45^{\circ}$ were used. Thirty-two scans with a resolution of $4 \mathrm{~cm}^{1}$ were obtained and averaged.

A morphological study of the PTFE surfaces after every treatment was achieved with a Philips XL-30 FEI EUROPE SEM microscope (Eindhoven, Holland). The samples were prepared using gold coating 


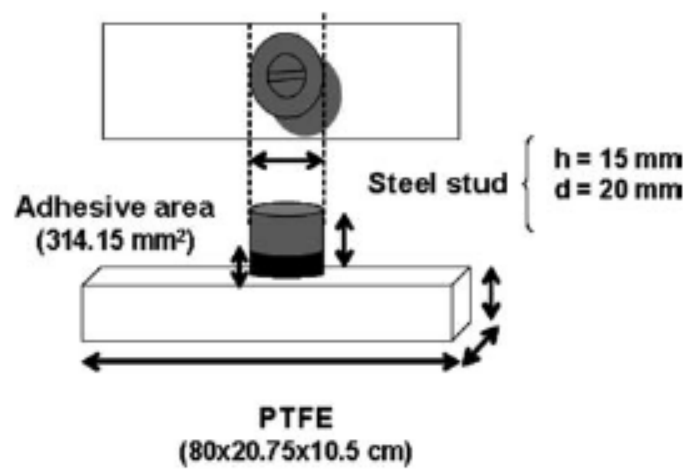

FIGURE 2 Scheme of the pull-off test specimen, including substrate (PTFE), adhesive, and stud.

in a Polaron ${ }^{n i g}$ high resolution sputter coater. The microscope was also equipped with a dispersive X-ray energy probe (EDX) which provided a qualitative atomic analysis of the surface.

\subsection{Pull-Off Test}

The adhesion properties both of the untreated and treated samples were tested using an Adhesion Tester KN-10 device (Neurtek Instruments, Eibar, Guipúzcoa, Spain) and following the UNE EN-24624 normative. As the device provides direct failure load values, it is necessary to transform them into tensile strength units:

$$
\sigma=\frac{F}{A}=\frac{F}{\pi \cdot r^{2}} .
$$

The steel studs (diameter $20 \mathrm{~mm}$ ) were adhesively bonded (Fig. 2) and allowed to cure under dust-free conditions $\left(25^{\circ} \mathrm{C}\right.$ and $\left.50 \% \mathrm{RH}\right)$ for 48 h. Polyurethane-based Sikaflex ${ }^{(16)}-252$ (Sika Corporation, Madison Heights, MI, USA) and epoxy Loctite ${ }^{(i)}$ Hysol $^{10} 9483$ A\&B (Loctite, Dublin, Ireland) adhesives were deposited onto the PTFE surfaces under each test condition. The acrylic Loctite $330^{\mathrm{TM}}$ (Loctite, Dublin, Ireland) was applied together with an activator Loctite -737 , deposited on the abraded and degreased steel studs, in order to obtain maximum adhesion. Six samples per studied condition were tested.

\section{RESULTS}

The first step of this investigation was the determination of pristine PTFE surface energy. As was expected, due to the chemical stability 
and the existence of fluorine atoms in the polymer backbone, PTFE yielded very high contact angle values (Fig. 1). Attending to the definition proposed by Young [12], contact angles larger than $90^{\circ}$ would not wet a surface, and are characteristics of low surface energy materials.

When the pre-treatments were applied, a more hydrophilic PTFE surface was observed. As can be inferred from Fig. 3, the use of chemical treatments increased total surface energy by $40-50 \%$, while APPT enhancement was located around $60 \%$. These results are in the same tendency as the ones presented by Liu et al. [13] when subjecting medical PTFE to long-distance $\mathrm{O}_{2}$ plasma treatment.

Due to the subsequent bonding process that PFTE is going to suffer, besides higher total surface energy, it is necessary to obtain a more polar surface appropriate to interact with adhesives. Results show that APPT practically did not change the polar fraction of the surface energy (from $0.53 \mathrm{~mJ} / \mathrm{m}^{2}$ of pristine surface to $0.88 \mathrm{~mJ} / \mathrm{m}^{2}$ ). This fact indicates that APPT activated PTFE, but it was energetically poor to achieve C-F bonding scission and introduction of nitrogen- or oxygencontaining functionalities, of a polar nature.

In contrast to this, all the studied wet chemical treatments led to approximately $90 \%$ higher values of the polar fraction. When PTFE surfaces were oxidised after APPT, this component, related to hydrogen bonds and van der Waals interactions, changed from 0.5 to $9.7 \mathrm{~mJ} / \mathrm{m}^{2}$. This effect was ascribed to the capability of APPT to activate PTFE due to radical reactions with plasma species, leading to

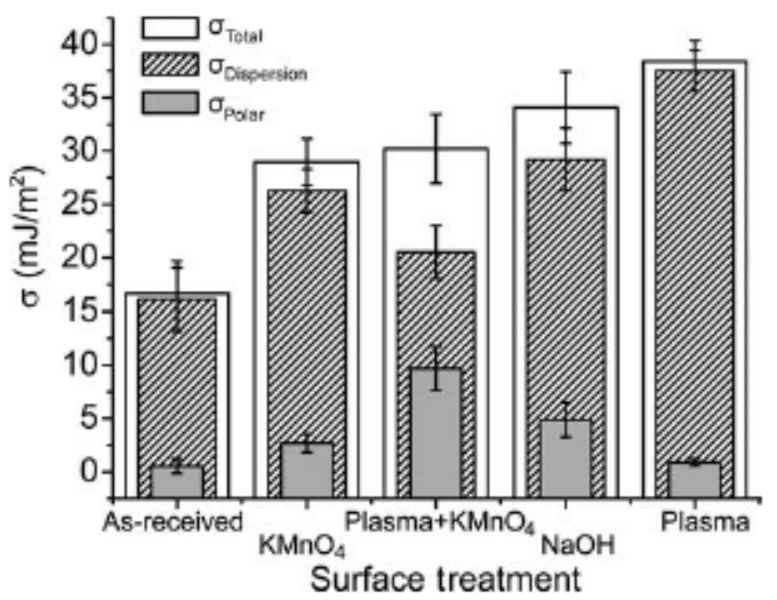

FIGURE 3 Variation of the surface energy components of PTFE with and without surface treatment. 
the generation of more active sites. The inmersion of APPT-treated samples in a strongly oxidising solution would yield higher polarity results than those of just APPT or oxidising due to the combined effects of active sites creation (APPT) and further reactions, including the introduction of oxygen (oxidation) in the previously described sites.

Results of surface energy after S180 abrasion are not shown because of their proximity to the ones of the as-received surfaces. As was previously described for other polymeric surfaces [14], the abrasion treatment yielded an improvement in adhesion, not because of a modification of the chemical composition but a creation of a great number of valleys and tops acting as sites of mechanical adhesion.

The SEM study of the samples is shown in Fig. 4. Pristine PTFE (Fig. 4a) presented a smooth topography, where lamination lines and fabrication heterogeneities, such as pores, could be observed. When the material was subjected to APPT (Fig. 4b), a rougher surface appeared, with homogeneous distribution of rounded micrometric (approximately $50 \mu \mathrm{m}$ ) structures. Similar features were observed after treatment with the $\left(\mathrm{KMnO}_{4} / \mathrm{HNO}_{3}\right)$ mixture (Fig. 4c), although the surface was modified to a lesser extent. When a combined process of APPT followed by oxidation was tested (Fig. 4d), in addition to the mentioned morphology, PTFE strands were found, which are assumed to emerge from the bulk material due to the treatment. Finally, the reaction with $\mathrm{NaOH}$ (Fig. 4e) led to the formation of holes, due to a deep depth attack, and the S180 abrasion (Fig. 4f) implied a pull-off of material and high rate of damage in the surface.

Regarding the surface energy and the SEM data, we could anticipate that APPT and oxidation processes were likely to create a more wettable PTFE surface by means of chemical composition modifications, while $\mathrm{NaOH}$ and $\mathrm{S} 180$ abrading would enhance mechanical interlocking of the substrate with adhesives through the generation of a more extensive anchoring area. As can be inferred from the EDX analysis results shown in Table 1, all the used treatments led to a reduction in the weight percentage of fluorine, to a larger extent when combining APPT and surface oxidation. It was also observed that a small quantity of oxygen and manganese appeared on the surfaces treated with the oxidising solution. These elements are easily attributed to the formation and deposition of brown $\mathrm{MnO}_{2}$ by the following reaction:

$$
2 \mathrm{KMnO}_{4}+2 \mathrm{HNO}_{3} \rightarrow 2 \mathrm{MnO}_{2}+2 \mathrm{KNO}_{3}+\mathrm{H}_{2} \mathrm{O}+\frac{3}{2} \mathrm{O}_{2} .
$$

FTIR-ATR analysis (Fig. 5) was used to check out the existence of new moieties formed in the PTFE samples, as well as to confirm the 

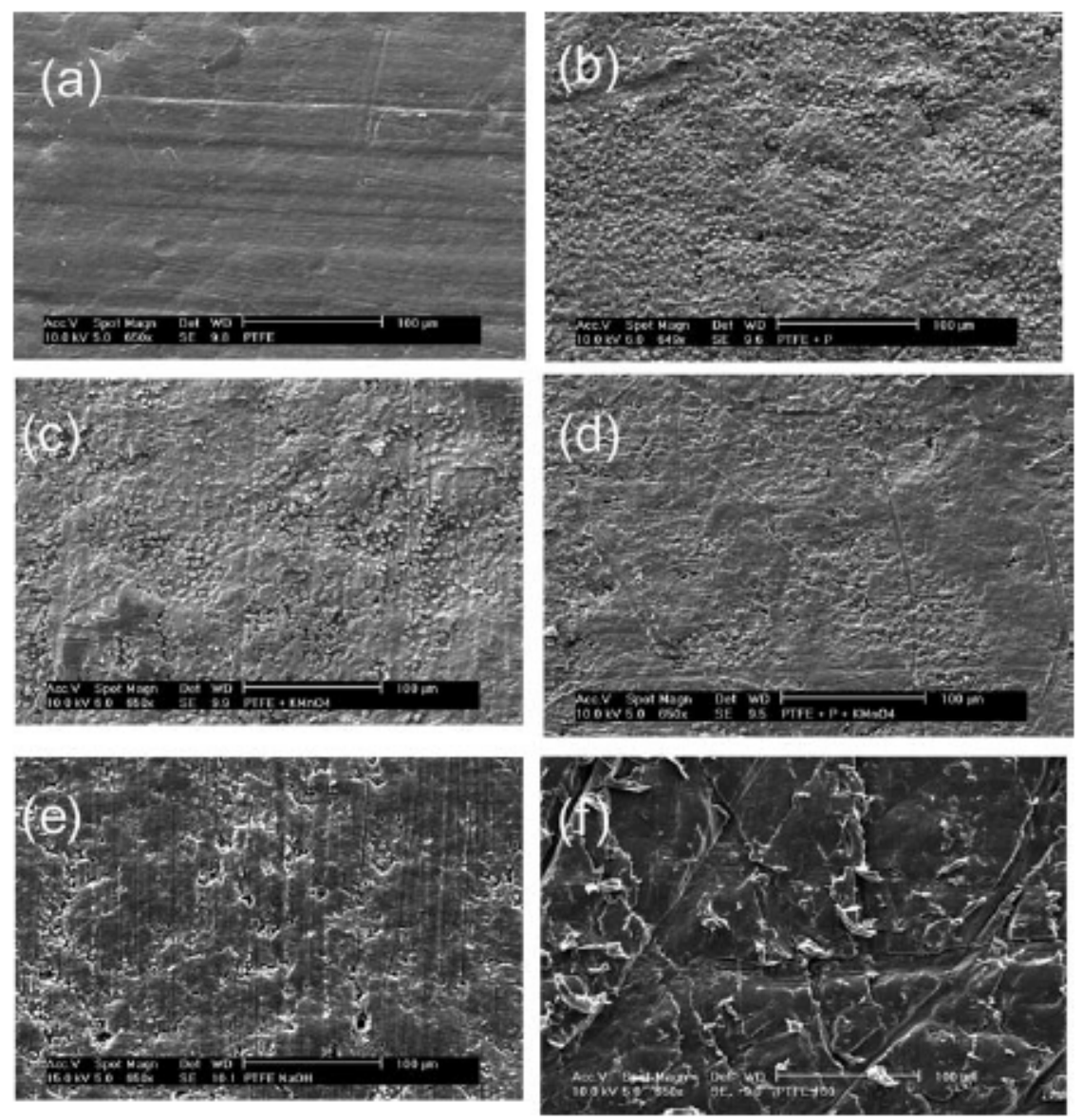

FIGURE 4 SEM micrographs of PTFE surface: (a) as-received, (b) plasma treated, (c) oxidised by $\mathrm{KMnO}_{4}+\mathrm{HNO}_{3}$, (d) plasma followed by chemical oxidation, (e) $\mathrm{NaOH}$-treated, and (f) abraded (180 grain size sandpaper).

TABLE 1 EDX Data of the Composition of the PTFE Samples Both Pristine and After the Surface Treatments

\begin{tabular}{lcccc}
\hline PTFE treatment & Wt.\% C & Wt.\% F & Wt.\% O & Wt.\% Mn \\
\hline Pristine & 24.1 & 75.9 & & \\
S180 abraded & 24.4 & 75.6 & & \\
APPT & 25.6 & 74.4 & & \\
NaOH & 29.2 & 70.8 & & \\
Oxidized & 18.7 & 67.2 & 5.5 & 8.6 \\
APPT +oxidation & 20.9 & 62.6 & 5.0 & 11.5 \\
\hline
\end{tabular}




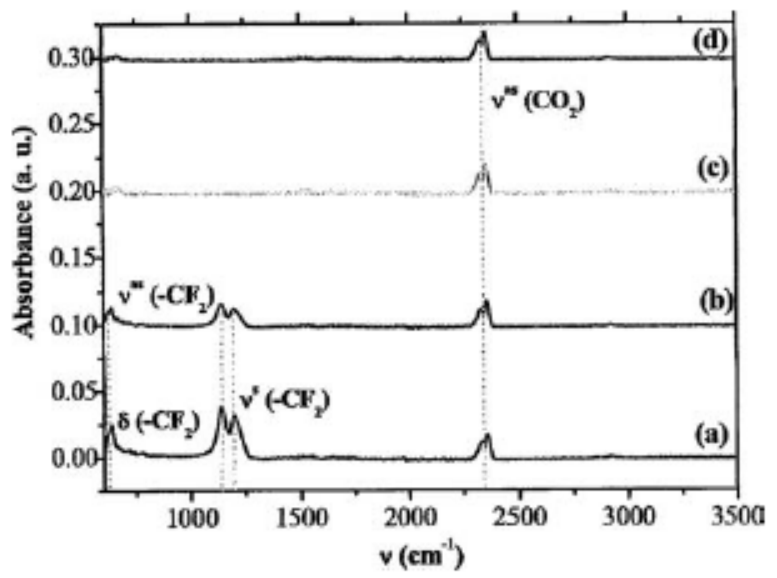

FIGURE 5 ATR-FTIR spectra of the surface conditions: (a) pristine, (b) plasma treated, (c) $\mathrm{NaOH}$, and (d) oxidised with the permanganate acid solution.

EDX results previously reported. Both bending and asymmetrical and symmetrical stretch $-\mathrm{CF}_{2}$ vibrations located at 637,1143 , and $1200 \mathrm{~cm}{ }^{1}$, respectively, disappeared in the infrared spectra of the oxidised and plasma treated samples, indicating that scission of C-F bonds took place. Although typical absorption bands of $\mathrm{C}=\mathrm{O}$ and $\mathrm{C}=\mathrm{C}$ groups (around 1760 and $1690 \mathrm{~cm}{ }^{1}$, respectively) were not observed in the infrared spectra, EDX results showed the existence of a small quantity of oxygen (approximately $5 \mathrm{wt} \%$ ) in the oxidised and plasma treated surfaces, as could be expected. The absence of this absorption band was attributed to the existence of carbonyl functionalities only on the outer surface layers, as other authors have reported [15].

Pull-off tests (Fig. 6) revealed an improvement of the adhesion properties of PTFE when subjected to surface treatments. Although experimental results presented larger errors, an improvement in adhesion is clearly observed due to the modifications induced by the processes used. In order to present a clear range of results, we are going to divide the adhesion experimental data attending first to the adhesive behaviour and finally to the effect of the treatment condition.

i. Compared with the acrylic Loctite 330 data, the Loctite Hysol 9483 A\&B epoxy-based adhesive showed better tensile strength values under all the pre-treatment conditions, varying from $15 \%$ for the untreated specimens, to $60 \%$ for the oxidised ones. The use of 


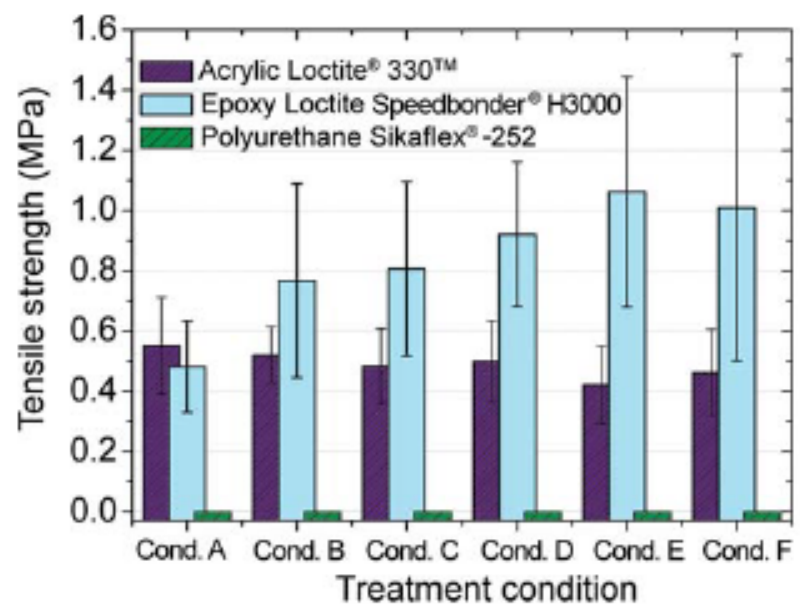

FIGURE 6 Pull-off results under the surface treatment conditions: Cond. A) untreated; Cond. B.) immersion in $\mathrm{NaOH}$; Cond. C) plasma; Cond. D) abraded; Cond. E) immersion in $\left[\mathrm{KMnO}_{4} / \mathrm{HNO}_{3}\right]$ and Cond. F) plasma followed by $\left[\mathrm{KMnO}_{4} / \mathrm{HNO}_{3}\right]$ (color figure provided online).

acrylic adhesive yielded not significant variations when PTFE was subjected to surface treatments, exhibiting values with a range of differences of $10-20 \%$, all of them falling into the calculated range of error. It was not possible to obtain valid pull-off experiment values of the joints with the polyurethane-based Sikaflex -252 adhesive, due to the easy and total debonding which took place in all the specimens.

ii. The assessment of the changes in tensile strength produced by surface pre-treatments is going to be mainly focused on the samples bonded with Loctite Hysol 9483 A\&B, which were the ones showing more remarkable variations. The analysis led to the conclusion that the most suitable process to prepare PTFE prior to bonding was the inmersion in $\left(\mathrm{KMnO}_{4} / \mathrm{HNO}_{3}\right)$, which was in agreement with the results of the surface energy polar fraction previously found. This treatment led to a tensile strength value of approximately $1.1 \mathrm{MPa}$, compared with the $0.5 \mathrm{MPa}$ found for the pristine surfaces. Results, considering the statistical error, were very similar for both surfaces firstly activated by APPT or just oxidised, differing by $5 \%$. As was previously described, it was not possible to accurately establish a most adequate treatment prior to bonding both with the acrylic- and the polyurethane-based adhesive, due to the small differences found for every sample and the impossibility to achieve adhesive bonding, respectively. 
Finally, we did not observe cohesive failure with PTFE under any of the test conditions. The best results were found for the oxidized samples, both APPT-activated and not, that showed mixed failure when bonding with an epoxy, which appears to be a promising result.

\section{CONCLUSIONS}

In this work, we study the improvement of the adhesion properties of PTFE by using different pre-treatments, namely: abrasion, chemical ( $\mathrm{NaOH}$ and $\mathrm{KMnO}_{4} / \mathrm{HNO}_{3}$ aqueous solutions), and APPT, with the main purpose of creating a more polar and wettable surface.

- APPT treatment led to the highest total surface energy data, approximately $94 \%$ higher than pristine PTFE.

- The polar component of the surface energy, related to hydrogen bonds and van der Waals interaction was increased to a higher extent when surfaces were oxidised after APPT activation. This fact was explained by the creation of more sites in the PTFE surface susceptible to achieve C-C and C-F scission by oxidation. Reactions would result in the introduction of oxygen and nitrogen, the polar nature of which would generate a more hydrophilic substrate. Defluorination and insertion of new atoms was confirmed by both FTIR-ATR and EDX analysis.

- SEM micrographs showed the creation of a similar topography for both APPT and oxidation, with a rougher surface, which seemed to be positive for adhesion due to the creation of more anchoring areas. $\mathrm{NaOH}$ and $\mathrm{S} 180$ abrasion treatments led to an important degradation of the surfaces, exhibiting the appearance of holes and pull out of material, respectively.

- Pull-off test results led to two main conclusions: in terms of adhesives, it was clearly demonstrated that the polyurethane based compound was not suitable to bond PTFE. The acrylic showed similar results, located around $0.5 \mathrm{MPa}$ of tensile strength, for all the conditions used, including pristine surfaces. However, epoxy performance was almost equal to acrylic when applied on as-received samples (strength of $0.48 \mathrm{MPa}$ for epoxy and $0.55 \mathrm{MPa}$ for acrylic), while results of treated PTFE bonded with epoxy were 50-55\% higher. As to the effect of the surface processes, the best results were achieved by oxidation, regardless of prior activation by APPT.

In conclusion, it has been proven that defluorination of PTFE surfaces, introduction of polar moieties, thus more hydrophilic, and creation of a rougher surface with larger anchoring area, could be 
obtained by subjecting samples to oxidation in $\left(\mathrm{KMnO}_{4} / \mathrm{HNO}_{3}\right)$. Proper adhesive bonding was achieved when using an epoxy-type material.

\section{REFERENCES}

[1] Dubbel, H., in Handbook of Mechanical Engineering, W. Beitz and K. H. Kuttner (Eds.) (Springer Verlag, Düsseldorf, 1994).

[2] Kang, E. T. and Zhang, Y., Adv. Mater. 12, 14811489 (2000).

[3] Mittal, K. L., Polymer Surface Modification: Relevance to Adhesion, (VSP/Brill, Leiden, 2009), Vol. 5.

[4] David, E., Lazar, A., and Armeanu, A., J. Mater. Process. Technol. 157 158, 284328 (2004).

[5] Kim, S. R. J., Appl. Polym. Sci. 77, 19131920 (2000).

[6] Allmer, K. and Feiring, A. E., Macromolecules 24, 5487 (1991).

[7] Wang, C. and Chen, J. R., Appl. Surf. Sci. 253, 45994606 (2007).

[8] Combellas, C., Ríchardson, S., Shanahan, M. E. R., and Thiébault, A., Int. J. Adh. Adh. 21, 5964 (2001).

[9] Badey, J. P., Espuche, E., Jugnet, Y., Chabert, B., and Duc, T. M., Int. J. Adh. Adh. 16, 173178 (1996).

[10] Wang, S., Li, J., Suo, J., and Luo, T., Appl. Surf. Sci. 256, 22932298 (2010).

[11] Encinas, N., Díaz Benito, B., Abenojar, J., and Martínez, M. A., Surf. Coat. Technol. 205, 396402 (2010).

[12] Fowkes, F. W., J. Phys. Chem. 67, 258 (1963).

[13] Liu, H., Zhang, H,, and Chen, J., Appl. Surf. Sci. 255, 81158121 (2009).

[14] Encinas, N., Pantoja, M., Abenojar, J., and Martínez, M. A., J. Adh. Sci. Technol. 24, 18691883 (2010).

[15] Caro, J. C., Lappan, U., and Lunkwitz, U., Surf. Coat. Technol. 116 119, 792795 (1999). 